\title{
Caecal adenocarcinoma with multiple synchronous small intestinal adenocarcinoma
}

\author{
J Lee, R Tamhane, TG Parks
}

\begin{abstract}
Summary
We describe a patient who presented with iron deficiency anaemia in whom both upper and lower gastrointestinal investigation were initially negative. Management included successive blood transfusions. Repeat investigation six months later revealed caecal adenocarcinoma. At laparotomy, the patient was also found to have two separate primary synchronous adenocarcinomas of the proximal small bowel. Right hemicolectomy and excision of the small bowel lesions were carried out. The patient eventually succumbed to the disease six weeks after discharge from hospital.
\end{abstract}

Keywords: adenocarcinoma, anaemia

Small bowel tumours are uncommon, notoriously difficult to diagnose and often advanced at the time of diagnosis. They often present with vague abdominal symptoms. Early discovery and appropriate surgical excision of the tumour provide the optimum outcome for the patient. Timely diagnosis depends on a high level of clinical suspicion and accurate radiological assessment of the small intestine.

We report a case of multiple small intestine primary adenocarcinomas with synchronous caecal cancer which presented with chronic blood loss and iron deficiency anaemia. The literature is reviewed with respect to small bowel adenocarcinoma, its presentation, treatment and prognosis.

\section{Case report}

A 78-year-old man was transferred from a District General Hospital for further management of persistent iron-deficiency anaemia. The patient had initially presented six months earlier with melaena and iron-deficiency anaemia. Oesophagogastroduodenoscopy had been carried out on two occasions without any positive findings. Flexible sigmoidoscopy to a distance of $45 \mathrm{~cm}$ from the anus had demonstrated diverticular disease. Barium enema did not reveal any other lesion and ultrasound scan of the abdomen was reported as normal. The patient was admitted on two further occasions to the District Hospital with iron-deficiency anaemia which was treated with blood transfusion.

On admission to our surgical unit, the patient was anaemic (haemoglobin $9.6 \mathrm{~g} / \mathrm{dl}$, packed cell volume 0.287 , mean corpuscular volume $80 \mathrm{fl}$ ), cachectic and dehydrated. Abdominal examination was normal apart from a scar from previous appendicectomy. Rectal examination revealed melaena. Sigmoidoscopy of the distal $15 \mathrm{~cm}$ of the large bowel did not show any mucosal lesion. Liver function tests were abnormal (alkaline phosphatase and $\gamma$ glutamyl transpeptidase were elevated to $1153 \mathrm{U} / 1$ and $225 \mathrm{IU} / 1$, respectively).

Repeat ultrasound scan of the abdomen showed no abnormality in the liver, spleen or pancreas but the scan disclosed a mass measuring $5.7 \times 5 \mathrm{~cm}$ between the spleen and upper pole of the left kidney, representing a possible lesion in the adrenal gland. Computed tomography confirmed the presence of a large soft tissue mass in the region of the left adrenal gland. There was no evidence of liver metastases or enlarged para-aortic or iliac lymph nodes. At colonoscopy, a large polypoid caecal lesion was detected. Histological examination of biopsies of this lesion confirmed the presence of poorly differentiated adenocarcinoma.

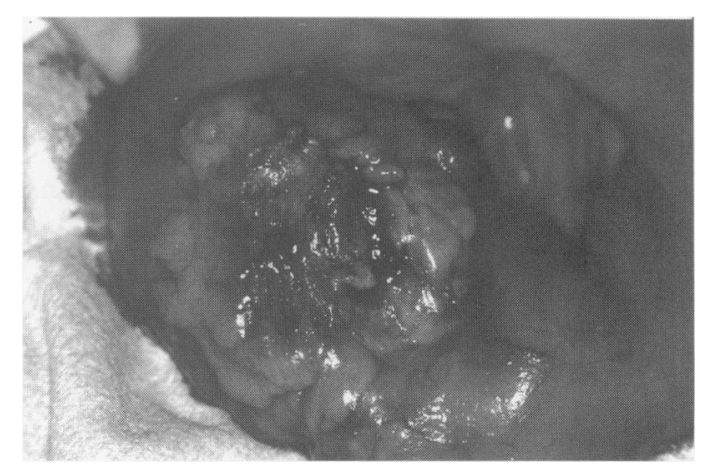

Figure 1

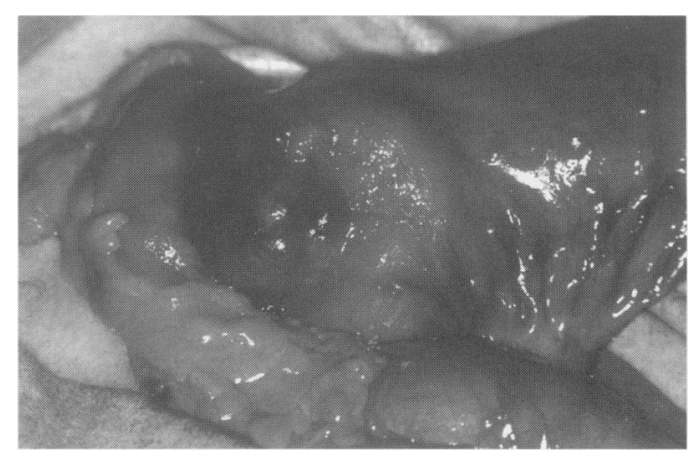

Figure 2 
Arrangements were made for elective right hemicolectomy for the caecal cancer to be performed. At the time of surgery, two further primary neoplastic lesions each measuring approximately $6 \mathrm{~cm}$ in diameter were discovered in the proximal small intestine. The first of these was situated $5 \mathrm{~cm}$ distal to the duodenal-jejunal flexure and the second was sited $30 \mathrm{~cm}$ more distally. There was no gross connection between the two tumours. The presence of a large fixed retroperitoneal mass in the left adrenal region was also confirmed at laparotomy. The liver, spleen and kidney were grossly normal. A segment of jejunum incorporating both tumours was resected and direct primary jejunal end-to-end anastomosis was undertaken. For the caecal carcinoma, right hemicolectomy was carried out with ileotransverse colic anastomosis. Postoperative recovery was uneventful but the patient remained weak and lethargic after the palliative procedure.

Histopathological examination showed that all three tumours were poorly differentiated adenocarcinomas with no attempt at gland formation. The tumour cells were large with vesicular nuclei, forming an infiltrative and sheeted pattern destroying and replacing the mucosa, submucosa and muscle coat extending into the serosa. Immunohistochemical stains again showed a poorly differentiated carcinoma. There was no evidence of Crohn's or coeliac disease in the adjacent small bowel mucosa. The regional lymph nodes showed reactive changes with no metastatic spread.

The patient was assessed by the clinical oncologist who felt that he was not medically fit for adjuvant chemotherapy. He was discharged home two weeks after surgery. His general condition gradually deteriorated and he died six weeks postoperatively.

\section{Discussion}

Small bowel malignancies are quite uncommon with an age-standardised incidence of around $1 / 100000^{1}$ in the US. They occur most frequently in the sixth and seventh decades of life with a male preponderance. ${ }^{2}$ Cancer of the small bowel accounts for $0.1-0.3 \%$ of all malignancies and $1-3 \%$ of all gastrointestinal malignancies. ${ }^{3}$ This is in spite of the fact that the small intestine accounts for $75 \%$ of the length and $90 \%$ of the surface area of the gastrointestinal tract. ${ }^{4}$ Adenocarcinoma of the small intestine is 40 to 60 times less common than its colonic counterpart. This relative resistance of small bowel mucosa to neoplastic changes is in part due to rapid transit time to carcinogens, alkalinity of the content, the higher level of hydrolases (thought to detoxify carcinogens) and a decreased bacterial count. There is also indirect evidence of an immunological protective mechanism. ${ }^{4}$

The most frequent symptoms associated with malignant small bowel tumours are abdominal pain (83\%), nausea and/or vomiting (54\%) and weight loss (43\%). Abdominal mass is the most common finding on physical examination (29\%), followed by distension
Small intestinal adenocarcinoma: clinical features and diagnosis

- nonspecific presentation with abdominal pain, nausea, vomiting and weight loss

- clinical examination will demonstrate abdominal mass in around $30 \%$ of cases

- occult gastrointestinal bleeding present in $50 \%$ of patients

- high index of suspicion in occult gastrointestinal bleeding with normal upper and lower gastrointestinal examinations

- spreads locally and to the regional lymph nodes

- small bowel adenocarcinoma may develop in patients with chronic, long-standing Crohn's disease

- most useful diagnostic tests are small bowel follow-through or small bowel enema examination

Small intestinal adenocarcinoma: management and treatment

- $50-80 \%$ of all small bowel tumours are amenable to surgical resection

- lymph node involvement results in reduction in 5-year survival

- radiotherapy and chemotherapy have not been found to be effective in advanced disease

- 5-year survival of small bowel adenocarcinoma is $15-20 \%$

(24\%) and abdominal guarding and rigidity $(14 \%){ }^{5,6}$ Our case presented with gastrointestinal bleeding which is encountered in $50 \%$ of patients ${ }^{4}$ while laboratory examinations are normal in $40 \%$ of patients. ${ }^{6}$ The average time from the onset of symptoms to diagnosis was $3.6-7.5$ months. ${ }^{5,6}$

The importance of a high index of suspicion and of the potential need for small bowel evaluation is emphasised by Donohoe. ${ }^{7}$ Failure to do so constitutes a common error when confronted.with occult gastrointestinal blood loss and normal upper and lower gastrointestinal examinations. Early diagnosis with prompt surgical resection remains the cornerstone of management that has been shown to improve prognosis. Lymph node involvement at the time of surgery results in significant reduction in five-year survival rate. Adjuvant combination chemotherapy has been used in advanced disease with occasional tumour regression but overall results from chemotherapy or radiotherapy are disappointing, ${ }^{1,4,7}$ and neither have been shown to improve prognosis significantly. ${ }^{1,4}$ Of the jejunal malignancies, $70 \%$ occur within the first $100 \mathrm{~cm}$ of the ligament of Treitz ${ }^{4}$; this location is exemplified in our case report. Adenocarcinoma was the most common histological pattern reported in several review papers. ${ }^{1,3,5,8}$

Although it is unusual to have multiple synchronous enteric and colonic malignancies as in our case, these have been recorded by Baillie et $a l^{6}$ and Garcia Marcilla et al. ${ }^{9}$ Neugut et $a l^{10}$ reported that the risk of colorectal cancer was increased following adenocarcinoma of the small intestine, and the risk of adenocarcinoma 
of the small bowel was also increased following colorectal cancer. Diagnosis of small bowel malignant neoplasms remains a difficult challenge owing to their nonspecific clinical presentation. Upper gastrointestinal endoscopy is excellent for diagnosis of proximal duodenal tumours but proper evaluation of the small intestine requires follow-through contrast examinations. In combination, these techniques reveal 50 to $70 \%$ of small bowel malignancies. ${ }^{4}$ Small bowel enema is an infrequently used radiological investigation to delineate and evaluate thoroughly the entire length of small intestine. ${ }^{1,4}$ This technique may occasionally identify lesions not demonstrable on small bowel follow-through series but it takes longer to perform, requires upper gastrointestinal intubation and is associated with prolonged radiation exposure. ${ }^{4}$ Most $(50-80 \%)$ of all

1 Goodman TR, Nolan DJ. Adenocarcinoma of the small intestine. Illustrated Case Report in Gastroenterology 1994; 1: intestin. $51-5$.

2 Sikorski T. Jejunal adenocarcinoma presenting as a lymphoproliferative disorder. Illustrated Case Report in Gastroenterology 1996; 3:45-8.

3 Matsuo S, Eto T, Tsunoda T, Kanematsu T, Shinozaki T. Small bowel tumours: an analysis of tumour-like lesions, benign and malignant neoplasms. Eur $\mathcal{F}$ Surg Oncol 1994; 20: $47-51$.

4 Aghazarian SG, Birly BC. Adenocarcinoma of the small intestine: a plea for early diagnosis. South Med F 1993; 86: $1067-9$.

5 Ciresi DL, Scholten DJ. The continuing clinical dilemma of primary tumours of the small intestine. Am Surg 1995; 61: primary small bowel tumours are amenable to surgical removal; the remainder being treated by palliative bypass to prevent or relieve intestinal obstruction. ${ }^{4}$ The prognosis of small bowel adenocarcinoma is poor, with a five-year survival of between $15-20 \% .^{1,2,4,9}$

In conclusion, it is important to recognise malignant small bowel tumour as a rare cause of vague abdominal symptoms and chronic unexplained gastrointestinal blood loss. When standard routine upper and lower gastrointestinal tract studies have been negative, further appropriate investigation on the small bowel may be indicated. Early diagnosis of small intestinal tumours with complete excision will lead to better long-term survival. Most authors recommend small bowel enema (enteroclysis) as the most appropriate and accurate form of investigative tool.

6 Garcia Marcilla JA, Sanchez Bueno F, Aguilar J, Parrilla Paricio P. Primary small bowel malignant tumours. Eur $f$ Purg Oncol 1994; 20: 630-4.

7 Donohue JH. Malignant tumours of the small bowel. Surg Oncol 1994; 3: $61-8$

8 Frost DB, Mercado PD, Tyrell JS. Small bowel cancer: a 30-year review. Ann Surg Oncol 1994; 1: 290-5.

9 Baillie CT, Williams A. Small bowel tumours: a diagnostic challenge. $\mathcal{F} R$ Coll Surg Edinb 1994; 39: 8-12.

10 Neugut AI, Santos J. The association between cancers of the small and large bowel. Cancer Epidemiol Biomarkers Prevent 1993; 2: 551-3. 